\title{
TRASTORNO POR DÉFICIT DE ATENCIÓN E HIPERACTIVIDAD, DIAGNÓSTICO, TRATAMIENTO Y POSIBILIDADES.
}

\section{ATTENTION DEFICIT HYPERACTIVITY DISORDER, DIAGNOSIS, TREATMENT AND POSSIBILITIES.}

\author{
Pablo Eugenio Castillo Armijo \\ Doctor en Educación y Sociedad \\ Universidad de Santiago de Chile, Santiago, Chile. \\ Académico del Departamento de Educación, Facultad de Humanidades, USACH. \\ pablo.castillo.armijo@gmail.com \\ Caterin Estefani Henríquez Sepúlveda \\ Licenciado de Educación \\ Universidad Católica del Maule, Talca, Chile \\ caterinhenriquez2804@gmail.com \\ Jasmin Verónica Hernández Aburto \\ Licenciado de Educación \\ Universidad Católica del Maule, Talca, Chile \\ jasminvhernandez@gmail.com \\ Valentina Andrea Montoya Castro \\ Licenciado de Educación \\ Universidad Católica del Maule, Talca, Chile \\ valentinamontoya557@gmail.com \\ Yazmin Del Rosario Salgado Lecaro \\ Licenciado en Educación \\ Universidad Católica del Maule, Talca, Chile \\ yazmin.1997.s@gmail.com
}

Resumen: El Trastorno por Déficit de Atención e Hiperactividad en los contextos educativos presenta un sobrediagnóstico en los estudiantes el que ha ido en aumento, lo que nos ha guiado a investigar sobre los procesos de evaluación y los posibles tratamientos de este mismo. Esta investigación está basada en una metodología de carácter cualitativodescriptivo, a través de una revisión bibliográfica. Se concluye que el diagnóstico para el TDAH es más clínico que pedagógico, donde generalmente se utilizan tratamientos a través de la medicación para regular la conducta de los estudiantes dentro del aula. Se reconoce la existencia de tratamientos alternativos a partir del uso de nuevas tecnologías y enfoques hacia el bienestar de los estudiantes, que están siendo implementados de forma incipiente en muchos países del mundo y del cual nuestro país debe aprender. 
Palabras Clave: TDAH, estrategias, innovación didáctica, orientaciones, diagnóstico.

Abstract: Attention Deficit Hyperactivity Disorder in educational contexts presents an overdiagnosis in students, which has been increasing, which has led us to investigate the evaluation processes and possible treatments of this disorder. This research is based on a qualitative-descriptive methodology, through a literature review. It is concluded that the diagnosis for ADHD is more clinical than pedagogical, where treatments through medication are generally used to regulate the behavior of students in the classroom. It recognizes the existence of alternative treatments, based on the use of new technologies and approaches to the welfare of students, which are being implemented incipiently in many countries of the world and from which our country should learn.

Keywords: ADHD, strategies, didactic innovation, orientations, diagnosis.

Resumo: 0 Transtorno de Déficit de Atenção e Hiperatividade em contextos educacionais apresenta um sobrediagnóstico nos alunos, que vem crescendo, o que tem nos orientado a investigar os processos de avaliação e possíveis tratamentos para o mesmo. Esta pesquisa baseia-se em metodologia qualitativa-descritiva, por meio de revisão bibliográfica. Conclui-se que o diagnóstico de TDAH é mais clínico do que pedagógico, onde os tratamentos geralmente são utilizados por meio de medicamentos para regular o comportamento dos alunos em sala de aula. É reconhecida a existência de tratamentos alternativos baseados no uso de novas tecnologias e abordagens para o bem-estar dos alunos, que estão sendo implementados de forma incipiente em muitos países do mundo e com os quais nosso país deve aprender.

Palavras chave: TDAH, estratégias, inovação didática, orientações, diagnóstico.

\section{INTRODUCCIÓN}

En los últimos años en Chile se han modificado y generado políticas públicas para incluir a la diversidad de estudiantes presentes en el sistema educativo nacional como lo es el Decreto $83^{\circ}$, (2015) sobre la diversificación de la enseñanza, que buscan asegurar una educación inclusiva y calidad para todos, propiciando la igualdad de condiciones y oportunidades, minimizando las barreras de aprendizaje y participación para todos los estudiantes en diferentes contextos escolares. (MINEDUC, 2015, p.12-14)

El sistema educativo chileno contempla dos modalidad en torno a la educación especial: se presentan centros educativos de educación especial que atienden a estudiantes con discapacidad sensorial, intelectual, motora, de relación y comunicación y trastornos específicos del lenguaje, y el Programa de Integración Escolar (PIE), que se implementa en los sistemas educacionales regulares, como una estrategia inclusiva, teniendo el propósito de favorecer la participación y el logro de los objetivos de aprendizaje en los estudiantes con 
Necesidades Educativas Especiales (NEE), permanentes y transitorias (MINEDUC, 2016, p. 9).

Chile cuenta con 2.027 escuelas especiales, 5.662 establecimientos escolares con PIE y 46 escuelas y aulas hospitalarias (Holz, 2018) en algunos centros educativos se implementó la denominada opción 4 de integración escolar que según el MINEDUC (2018, p.32) consiste en un curso compuesto por estudiantes que presentan un diagnóstico asociado a NEE de tipo permanente. Según la base de datos del Ministerio de Educación de Chile (MINEDUC), el año 2018, había 183.373 alumnos matriculados que presentan NEE diagnosticados con discapacidad auditiva, discapacidad intelectual, discapacidad visual, trastorno del lenguaje, trastornos motores, autismo, alteraciones en la capacidad de relación y comunicación, trastorno por déficit de atención e hiperactividad.

La opción 4 de integración escolar consiste cuando los estudiantes que presentan un diagnóstico asociado a NEE de tipo permanente o transitorio asisten a todas las actividades en el aula de recursos y comparten con los estudiantes del establecimiento común, en recreos, actos o ceremonias oficiales del establecimiento o de la localidad y actividades extraescolares en general (MINEDUC, 2018, p. 32).

El PIE representa el 5,12\% de la matrícula total nacional de educación regular (Holz, 2018 , p. 1), datos que han crecido exponencialmente en los últimos años, por lo que la mayoría de los establecimientos educacionales chilenos cuentan con PIE, donde se realizan actividades de coeducación, a través del trabajo conjunto entre docentes regulares y profesionales de apoyo, entre ellos, profesores de educación especial, psicólogo, kinesiólogo, terapeuta ocupacional, asistente social, entre otros, generando ambientes de trabajo colaborativo en las distintas actividades pedagógicas, como la planificación, organización y co-enseñanza entre los docentes y los profesionales del PIE.

Debido a esto, las escuelas se encuentran obligadas a implementar diversas estrategias que favorezcan la participación y el logro de los aprendizajes esperados de todos los estudiantes en la sala de clases, potenciando las innovaciones educativas para dar respuesta a las diversas formas de aprender (MINEDUC, 2016).

Investigaciones señalan que el desarrollo profesional de los profesores en muchas ocasiones es insuficiente en relación a la generación e implementación de didácticas y estrategias para atender NEE de los alumnos en la sala de clases, ya que la formación no profundizan en conocimiento sobre estrategias y didácticas, centrado sus actividades a la hora de planificar en los alumnos "promedio", ignorando la diversidad de una sala de clases y las múltiples formas de aprender (Tenorio, 2011, p. 263).

De los alumnos con NEE integrados en el PIE, la presente investigación se enfocará en aquellos integrados en enseñanza básica diagnosticados con trastorno de atención e hiperactividad (TDAH), destacando las múltiples estrategias implementadas en el aula. 


\section{MARCO TEÓRICO}

\section{Definición del Trastorno por Déficit de Atención e Hiperactividad (TDAH)}

Una de las principales caracterizaciones que define los criterios que se presenta el TDAH son los impuestos por el Manual Diagnóstico y Estadístico de los Trastornos Mentales (Diagnostic and Statistical Manual of Mental Disorders, DSM) de la Asociación Americana de Psiquiatría (American Psychiatric Association, APA).

El TDAH, según el DSM-5 (APA, 2013, p. 33) es un trastorno de origen neurológico, caracterizado por una inatención persistente y/o hiperactividad-impulsividad que interviene con el funcionamiento o el desarrollo. Este comportamiento se da en más de un contexto o situación (hogar, escuela u otro) y afecta las relaciones con su entorno familiar, social y educativo.

En Chile, en el decreto $\mathrm{N}^{\circ} 170$ del 2009 (MINEDUC, 2009a), el Trastorno de Déficit Atencional o Trastorno Hipercinético, es definido como un:

[...] trastorno de inicio temprano, que surge en los primeros 7 años de vida del o la estudiante y que se caracteriza por un comportamiento generalizado, con presencia clara de déficit de la atención, impulsividad y/o hiperactividad. Este comportamiento se evidencia en más de un contexto o una situación, tales como el hogar, la escuela y/o actividades sociales, entre otras, y produce un malestar clínicamente significativo o una alteración en el rendimiento social o académico del o la estudiante. (p. 15)

Investigaciones señalan que la incidencia de diagnósticos es de un 3\% a un 7\%, siendo considerado a nivel mundial uno de los trastornos del neuro-desarrollo más frecuentes en población infantil y adolescente (Peña, Palacio, \& Barragán, 2010, p. 95).

En la Declaración de Cartagena para el Trastorno por Déficit de Atención con Hiperactividad del 2010 (TDAH) (Peña, Palacio, \& Barragán, 2010, p. 95), se menciona que en Latinoamérica existen al menos 36 millones de personas con TDAH y menos de un cuarto de los pacientes se encuentran bajo tratamiento y/o apoyo adecuado contando solo el 23\% tiene con un apoyo terapéutico psicosocial y el $7 \%$ con un tratamiento farmacológico adecuado.

En relación a su origen neurológico, aunque se han realizado estudios genómicos en pacientes con TDAH, aún no existen candidatos genéticos seguros, aun así se han encontrado algunas posibles regiones cromosómicas comunes entre pacientes, viéndose afectados genes que regulan los mecanismos de liberación de neurotransmisores; concretamente en los receptores y transportadores de catecolaminas (adrenalina, noradrenalina y dopamina), lo que explicaría las conductas de estas personas, como inatención, impulsividad y/o hiperactividad motora (Muñoz, et al., 2019, p. 38). 
El TDAH es un trastorno que presenta alta comorbilidad con otros trastornos, $67 \%$ de los estudiantes con TDAH presentan entre 1 o 2 diagnósticos adicionales, siendo los más frecuente el Trastorno del Desarrollo de Coordinación Motora, el Trastorno Oposicionista Desafiante, Trastorno Específicos del Aprendizaje y Trastorno Específico del Lenguaje (Varía y Kleinsteuber, 2014, p. 21)

Este trastorno se puede presentar en 3 subtipos descritos en el DSM-5, que apoya y orienta la detección y orientación de estudiantes con TDAH:

I. Subtipo con predominio del déficit de atención: El subtipo con predominio en el déficit de atención es más difícil de identificar. Suele pasar desapercibido, ya que no presenta problemas de conducta que interfieren en la actividad escolar, familiar o social, por lo general son aquellos estudiantes que se distraen fácilmente y presenta un periodo corto de atención, esto puede generar fracaso escolar durante los primero años de estudios, ya que no son capaces de seguir las demandas de organización y planificación propias en sus etapa educativa lo que crea lagunas acumuladas y por la falta de hábitos de estudio (Balbuena, et al. 2014, p. 16).

En el documento Protocolo para la Detección y Evaluación del Alumnado con Trastorno por Déficit de Atención e Hiperactividad en el Ámbito Educativo (en adelante protocolo TDAH). (Balbuena, et al. 2014, p. 16) indica que los estos estudiantes suelen presentar: Dificultades para reorientar su atención y para mantenerla de modo sostenido, tanto en el trabajo como en el juego/ Cuanto más monótona o compleja es la actividad más dificultades de atención presentan/ Dificultades en la realización de actividades que exigen un procesamiento secuencial/ Dificultades para prestar atención simultáneamente a dos actividades/ En actividades que son de su interés, la capacidad de atención se incrementa significativamente, de ahí que con frecuencia la familia y los profesores piensen que se trata de fallos en la motivación/ A menudo cometen errores por ignorar los detalles/ Con frecuencia parecen no oír cuando se les llama/ Con frecuencia no siguen las instrucciones y fracasan en la finalización de las tareas y de los juegos/ A menudo tienen problemas para organizar las tareas y las actividades/ Abandonan frecuentemente las actividades que requieren esfuerzo mental/ Constantemente pierden cosas que necesitan/ Con frecuencia se distraen con cualquier estímulo/ Dificultades con el manejo y control del tiempo.

II. Subtipo con predominio hiperactivo-impulsivo: Los estudiantes con este subtipo suele llamar la atención de la familia y de los profesores, ya que la impulsividad le genera dificultades para pensar antes de actuar y por ello interrumpen con frecuencia, además presentan hiperactividad lo que ocasiona que estén en constante movimiento (Balbuena, et al. 2014, p. 17).

En torno a las conductas de impulsividad el Protocolo de TDAH (Balbuena, et al. 2014, p. 18) menciona que : A menudo se le escapan las respuestas antes de terminar las preguntas, aparentemente dicen lo primero que se les ocurre/ Tienen dificultades para esperar su turno/ Con frecuencia interrumpen o se entrometen en las conversaciones de los demás/ Aunque conozcan las reglas, e incluso puedan explicarlas, son incapaces de pararse a pensar 
en las consecuencias antes de actuar/ Con frecuencia reciben castigos de su familia y profesorado porque consideran que no siguen las normas de forma intencionada/ Obtienen poco provecho de sus experiencias.

Las principales conductas de la hiperactividad son: Se mueven o se retuercen de forma nerviosa/ Con elevada frecuencia se levantan de la silla/ A menudo corren o saltan cuando no deberían; en adolescentes y adultos puede limitarse a sentimientos subjetivos de inquietud/ Con frecuencia tienen dificultades para hacer tranquilamente actividades de trabajo y de ocio/ Siempre están en movimiento continuo, como si tuvieran un motor/ Hablan excesivamente/ En adultos con TDAH la hiperactividad suele manifestarse como sentimientos de inquietud física.

III. Subtipo combinado: En este subtipo se presentan conjuntamente las conductas hiperactivas-impulsivas y las conductas de inatención (Balbuena, et al. 2014, p. 19). Algunas de las conductas más frecuentes del subtipo combinado sacados del Protocolo para la Detección y Evaluación del Alumnado con Trastorno por Déficit de Atención e Hiperactividad en el Ámbito Educativo del (Balbuena, et al. 2014, p.19-20) son: No pueden prestar atención concentrada durante mucho tiempo/Con frecuencia se sienten nerviosos, sensibles o tensos/ Tienen dificultad para estar quietos/ Exigen mucha atención de las personas de su entorno/ No cumplen las indicaciones y normas en casa/ Hacen las cosas sin pensar/ Les gusta llamar la atención/ No hacen o hacen incorrectamente las tareas escolares/ Presentan fuertes brotes de ira, rabietas o llanto/ Se enfadan con mucha facilidad/ Se muestran testarudos, mentirosos y tramposos/ Hacen ruido, gritan mucho/ Hablan en exceso/ A veces parece que no escuchan o no comprenden/ Prefieren jugar con niños y niñas más pequeñas.

\section{Procesos de evaluación del TDAH}

Debemos tener en cuenta que el diagnóstico de este trastorno no es realizado por profesionales de la educación, si no que éste es realizado por profesionales de la salud los que varían según las normativas de cada país, generalmente son neurólogos y psicólogos, debido a sus origen neurológico antes mencionando, por los que todas estas manifestaciones o conductas, no nos permite diagnosticar el TDAH, pero sí nos pueden prevenir de la presencia de estudiantes con este trastorno, por lo que debemos estar atentos a cualquier señal, cuando se tienen sospechas de la presencia de alumnos con TDAH se da inicio a un proceso de evaluación psicopedagógica que según el artículo 45 del Decreto 170 (2009, p. 17) la evaluación diagnóstica debe considerar un proceso de detección y derivación y un proceso de evaluación diagnóstica integral el que comprende la valoración de: anamnesis, evaluación pedagógica integral por el profesor de aula, entrevista a la familia, revisión de antecedentes escolares, aplicación de pruebas en base a criterios como el Test de Conners. Las escuelas que cuentan con el "Programa Habilidades para la Vida" pueden aplicar el cuestionario TOCA-RR para profesores y el cuestionario PSC para padres, entre otros; esta evaluación integral debe considerar la evaluación médica, psicopedagógica y la información proporcionada por el contexto escolar y familiar, recogida en el proceso de detección. 
Para llevar a cabo la evaluación diagnóstica integral el Ministerio de Educación ofrece diversos instrumentos de recogida de datos, pero también se pueden aplicar otras pruebas y entrevistas que apoyen este proceso, se detallan a continuación algunas herramientas de valoración para la detección y diagnóstico de estudiantes con TDAH.

\section{Entrevistas con la familia:}

Este proceso es muy relevante, ya que la familia puede entregar información de gran importancia en la entrevista acerca de las características, las posibles causas, la evolución y la repercusión del trastorno en la vida del niño o niña, y de sus familiares, interés de la familia por solucionar el problema y de las expectativas que tienen con respecto a su hijo o hija y sobre las posibles soluciones (Balbuena, et al., 2014, p. 37) para este proceso debemos tener en cuenta que se debe expresar con total claridad que el objetivo de la entrevista, buscando establecer una relación de colaboración entre la familia y la escuela, y orientar a la familia en los apoyos que requiere el estudiante.

El protocolo para la detección y evaluación del alumnado con trastorno por déficit de atención e hiperactividad en el ámbito educativo (Balbuena, et al. 2014, p. 39) nos menciona 2 entrevistas estructuradas y semiestructuradas las que han demostrado buenos resultados:

a) Entrevista semiestructurada; Clinical interview for child and adolescent ADHD patients (Barkley, 2006) dirigida a familias de niños y niñas con TDAH, contiene 6 secciones que recogen la historia del desarrollo, la historia médica, la historia de tratamientos anteriores, la historia escolar, la historia familiar y la historia social del niño o niña.

b) Entrevista diagnóstica para niños, niñas y adolescentes; Diagnostic interview for children DICA-P (Reich, Shayka, Taiblenson, 1988), tiene el objetivo de obtener información detallada y fiable sobre la conducta del niño o niña, consta de 247 ítems que exploran los diversos síntomas de alteraciones conductuales, tales como trastorno por déficit de atención con hiperactividad, trastorno negativista-desafiante, trastorno disocial, ansiedad de separación, etc. La valoración de la escala recoge la ausencia, la presencia y la duración de los síntomas de los trastornos.

\section{Cuestionarios para familias y profesores}

Al igual que la información que nos puede entregar las familias, es de gran importancia también conocer la percepción de los profesores, para esto se sugiere aplicar se aplican cuestionarios y escalas de conductas, los que deben ser aplicados a más de una persona cercana al niño en más de 2 contextos diferentes, para tener una visión general de los problemas de los estudiantes (Balbuena, et al., 2014, p. 39). 
Algunos cuestionarios y escalas de conducta que pueden servir al profesional para el diagnóstico del TDAH que nos presentan en el protocolo para la Detección y Evaluación del Alumnado con Trastorno por Déficit de Atención e Hiperactividad en el Ámbito Educativo (Balbuena, et al., 2014, p. 40) son:

a) CPRS-R: Conners' Parent Rating Scale-Revised para familias (Conners 1997a). CTRSR: Conners' Teacher Rating Scale-Revised para el profesorado (Conners, 1997). Se trata de un cuestionario que puede ser contestado por la familia (CPRS-R) y/o los profesores (CTRS-R) de niños y niñas entre los 3 y los 17 años, con el fin de valorar la presencia y severidad de conductas relacionadas con el TDAH. Está compuesto por cuatro factores: oposicionismo, desatención, hiperactividad-impulsividad e índice del TDAH. Por lo general se aplican la versión abreviada para la familia que contiene 27 ítems y la versión abreviada para los profesores que contiene 28 ítems, los que se agrupan en las subescalas de problemas de conducta, hiperactividad, desatenciónpasividad e índice de hiperactividad.

b) SNAP-IV-R: Rating Scale-Revised. SNAP-IV: Teacher and Parent Ratings Scale (Swanson, 2003). Este instrumento es completado por la familia y el profesor de niños entre 6 y 18 años, contiene 90 ítems, donde se exponen síntomas de TDAH y también del trastorno oposicionista desafiante (TOD) y de agresividad. Existen diferentes modificaciones de esta escala, pero nos centraremos en la escala SNAP-IV que incluye 18 ítems del TDAH y 8 ítems del trastorno oposicionista desafiante, síntomas especificados en el DSM-IV (APA, 1994) y CIE-10 (clasificación de los trastornos mentales y del comportamiento).

\section{Evaluaciones al alumnado}

Esta etapa del proceso de evaluación psicopedagógica no está directamente relacionada con el diagnóstico del TDAH, pero sí nos permite conocer las fortalezas y debilidades que presentan los estudiantes debido al trastorno, gracias a la recopilación de datos que integra información de las habilidades, competencias, estilos y dificultades de aprendizaje, los diferentes tipos de atención, el desarrollo de las funciones ejecutivas, aspectos socioemocionales (Balbuena, et al., 2014, p. 44). Estas evaluaciones varían de acuerdo al país en el que se encuentra el alumno, pero se recomienda que se evalúen todas las áreas anteriormente mencionadas para obtener una visión general del estudiante. Algunas evaluaciones estandarizadas son: WISC-IV: Escala de inteligencia de Wechsler para niños y niñas/ TEA: Test de aptitudes escolares/ EVAMAT: Prueba para la Evaluación de la Competencia Matemática/ EVALEC: Prueba para la Evaluación de la Competencia lectora/ CSAT: Tarea de atención sostenida en la infancia (Servera y Llabrés, 2004) / PROLEC y PROESC: Evaluación de los procesos lectores (Cuetos, Rodríguez y Ruano, 1996) / TALE: Test de análisis de lectoescritura (Toro y Cervera, 1990) 


\section{Tratamiento médico del TDAH}

En Chile y el mundo, se ha generado una discusión social entorno al diagnóstico y el uso de fármacos en el tratamiento de TDAH, el gran aumento en los diagnósticos de TDAH y el consumo de medicación en niños y niñas a nivel global ha provocado fuertes debates y controversias en torno a la validez del diagnóstico, la explicación de sus causas y formas de tratamiento (Reyes, Cottet, Jimenez y Jauregui, 2019, p. 41).

En torno a esta discusión se observan 2 miradas opuestas; aquellos que sospechan que el diagnóstico del TDAH está bajo la influencia de la industria farmacológica y por otro lado están quienes enfatizan en su origen neurológico o genético (Reyes, et al., 2019, p. 40).

El tratamiento del TDAH pueden producir efectos emocionales de alivio y mejora del rendimiento escolar, ya que, reducen la hiperactividad y la impulsividad y mejoran su capacidad para concentrarse, trabajar y aprender; pero también puede generar experiencias de miedo y efectos negativos sobre la autoestima y el sentido de control sobre la propia vida, algunos cuidadores de señalan que el TDAH es generalmente el malestar escolar (Reyes, et al., 2019, p. 42) y que el tratamiento no necesariamente podrían requerir una intervención psicológica, biomédica o farmacológica, lo que buscan las escuelas es acercar al ideal de normalidad y autocontrol gracias a la medicalización, pero perdiendo el sentido de autonomía de los alumnos (Reyes, et al., 2019, p. 51).

Los diferentes países en el mundo han desarrollado a través del tiempo, diversas estrategias para atender esta necesidad educativa especial, desencadenando opiniones tanto divididas como afines. Es así como en Estados Unidos el TDAH se trata comúnmente con medicamentos, educación o capacitación, terapia o una combinación de tratamiento.

En el contexto latinoamericano, específicamente en Brasil, El Hospital Sant Joan de Déu Barcelona, cuenta con una unidad especializada en casos graves de trastorno de déficit de atención e hiperactividad, en donde se han ido desarrollando nuevas estrategias de apoyo y atención a las personas con este trastorno.

En España, se encuentra la Federación Española de Asociaciones de Ayuda al Déficit de Atención e Hiperactividad (FEAADAH), una asociación sin fines de lucro que, instaurada en el año 2002, promueve la investigación del TDAH y colabora en áreas educativas, de atención social y científicas, con entidades consagradas al estudio y atención de este trastorno.

La primera línea de tratamiento para el TDAH son los estimulantes, reducen la hiperactividad y la impulsividad y mejoran su capacidad para concentrarse, trabajar y aprender. Muchos investigadores piensan que los estimulantes son efectivos porque el medicamento aumenta la dopamina, una sustancia química del cerebro, que juega un papel esencial en el pensamiento y la atención (National Institutes of Health, 2016). 
Realizar terapia junto a un plan de tratamiento para el TDAH puede ayudar a los pacientes y sus familias a enfrentar mejor los desafíos diarios, los profesionales de la salud desarrollan programas para educar a los padres o cuidadores de un niño con TDAH sobre el trastorno y cómo este afecta a su entorno, además de ayudar al niño y a sus padres a desarrollar nuevas habilidades, actitudes y formas de relacionarse (National Institutes of Health, 2016).

Similar al contexto norteamericano es el Reino Unido, donde se establece que en edad preescolar no se puede optar por tratamiento farmacológico, los especialistas de la salud deben derivar a un programa de formación y educación a los padres o cuidadores de los niños con TDAH como primera opción de tratamiento. El tratamiento farmacológico no es indicado como primera medida en niños y jóvenes con TDAH, este es reservado sólo para aquellos casos en que se presentan síntomas graves, que ya hayan experimentado intervenciones alternativas no farmacológicas sin respuestas positivas a aquellos tratamientos o cuyos síntomas no tengan respuesta a los programas de formación para padres o al tratamiento psicológico en grupo. (National Collaborating Centre for Mental Health UK, 2009).

En su contra parte se encuentra una postura de profesionales, con tendencias más bien psicoanalíticas, que pone en duda dicha patología y el tratamiento de la misma enfocado en la regulación neurológica, negando su base en lo fisiológico (Barceló, et al, 2018), ya que, se asocia el tratamiento farmacológico como mecanismo regulador de la conducta infantil. Si bien, los entrevistados han afirmado que en casos extremos o de sufrimiento explícito, es útil una evaluación para sumar un procedimiento medicalizado, sostienen que este tendría que ser el último recurso para implementar dentro del tratamiento, lo opuesto a lo que normalmente sucede.

Junto con los diagnósticos apresurados, colaboran a crear en el niño una representación de sí mismo negativa y a estigmatizar con una identidad social patologizante, que será difícil cambiar con el tiempo y que fomentará el aislamiento, convirtiéndose en una etiqueta que lo segrega (Barceló, et al, 2018). Si la medicalización se viera como algo natural y la implementación de esta como mecanismo base del tratamiento, corremos el riesgo de homogeneizar a los estudiantes sin tomar en cuenta el grado de sus síntomas y sus características individuales, catalogando conductas como positivas y negativas, y leyendo la no adaptabilidad escolar, pudiendo arriesgar a los alumnos en futuros problemas por los efectos adversos producidos por el uso excesivo de medicamentos (Barceló, et al, 2018).

\section{Nueva pedagogía para la atención del TDAH}

Existe una amplia gama de estrategias pedagógicas destinadas a atender el TDAH, así como otros trastornos con síntomas similares que han demostrado dar buenos resultados, sin embargo, se debe considerar que estas estrategias y terapias alternativas son

complementarias al uso de fármacos debidamente recetados bajo la evaluación y diagnóstico 
médico, bajo esta afirmación se han desarrollado diversas terapias y apoyos para mitigar las conductas disruptivas y/o negativas presentes en el alumnado con TDAH. emocional:

Encontramos en primer lugar todas las estrategias basadas en el tratamiento

a. Equinoterapia:

La terapia con animales específicamente la que se realiza con caballos, es denominada equinoterapia se ha ido desarrollando con diversos objetivos por el transcurso de los años, consiste en un trabajo en conjunto de profesionales competentes guiando al animal en las sesiones realizadas con los pacientes, tiene el fin de generar una mejora en aspectos, físicos, psicológicos, sociales, mentales y emocionales en los pacientes. El método horse emotion es un tratamiento que se desarrolla en un entorno pleno de estímulos sensoriales, sociales y educacionales. Este ambiente en el que transcurre la sesión, estimulante y motivador y fuera del ambiente escolar, sirve para enseñar al usuario y ayudarle para que desarrolle habilidades cognitivas en varios ámbitos (Eraud, 2020, p. 114).

Este método se llevó a cabo en un estudio cuya población se componía por estudiantes diagnosticados con TDAH de la ciudad de Barcelona (Cataluña), en el que se establece una evaluación inicial el cuestionario psico-emocional, de comunicación y socialización adaptación Roche (2017) modelo de EPONA (Eraud, 2020, p. 113).

El método horse emotion considera un trabajo interdisciplinar el contacto con el caballo representa un contexto propicio para la enseñanza de las técnicas de mindfulness y la práctica del yoga encima del caballo (Eraud, 2020, p.114).

\section{b. Mindfulness:}

El mindfulness es una terapia basada en actividades realizadas a través de ejercicios de relajación, respiración, y la noción de conciencia de ello Kabat-Zinn, 2003, en López y Pastor (2019, p. 145) presentan este término como "el estado particular de conciencia que surge al poner la atención, de forma intencional y sin juzgar, en el momento presente, viviendo la experiencia momento a momento".

Huguet y Alda, existe evidencia preliminar de que el mindfulness puede ser útil para los síntomas nucleares del TDAH y los déficits en las funciones ejecutivas (2019, p. 21)

Existen diversos beneficios de la implementación de terapia a través del mindfulness García-Campayo, 2018, citado por Huguet y Alda, (2019, p. 15) exponen que, mejora la capacidad para mantener la atención y concentración, disminuye la impulsividad, aumenta la tolerancia a la frustración, ayuda a calmarse y relajarse, potencia la identificación de sensaciones corporales, emociones y pensamientos, favorece la regulación emocional, promueve la introspección y facilita el autoconocimiento, mejora las relaciones interpersonales, reduce el estrés y el malestar psicológico y mejora la calidad de vida. 
En los contextos educativos según López y Pastor (2019), la práctica del mindfulness podría ayudar al alumnado a reducir el estrés y aumentar su propio bienestar, para alcanzar y optimizar el aprendizaje y mejorar sus relaciones interpersonales.

Este tratamiento alternativo ha sido analizado por diferentes autores en los contextos educativos. López y Pastor (2019), afirman que las intervenciones basadas en la atención plena (mindfulness) en las escuelas se evidencian como una alternativa para la mejora de la auto-regulación emocional y atencional del alumnado, favoreciendo disposiciones prosociales, su adaptación al medio y logró, por tanto, de un mayor bienestar psicológico.

\section{c. Musicoterapia:}

Las terapias alternativas se desarrollan a través de diferentes materias, una de ellas es la música, que según Rodríguez (2019, p. 42) la musicoterapia es la utilización de la música y / o de sus elementos (sonido, ritmo, melodía y armonía), por un musicoterapeuta calificado, con un cliente o grupo, en un proceso sistematizado para facilitar y promover la comunicación, la relación social, el aprendizaje, la movilización, la expresión, y la organización de procesos psíquicos u otros objetivos terapéuticos relevantes para satisfacer las necesidades físicas, emocionales, cognitivas y sociales.

Peñalba (2010), citado por Acebes y Carabias, (2014, p. 4) expone que:

Además de atenuar los síntomas de desatención y de falta de concentración, la música, a su vez, también repercute en una mejora de las relaciones sociales y la comunicación, siendo el medio idóneo para expresar y canalizar las propias emociones. La música aumenta la tolerancia a la frustración y disminuye la emisión de conductas disruptivas. Algunos niños con TDAH tienen dificultades para entablar y mantener relaciones con sus compañeros, debido principalmente a sus conductas impulsivas ( $p$. 4).

En estudiantes con TDAH, la utilización de esta terapia alternativa incide positivamente en diferentes procesos (Rodríguez,2019, p. 23) expresa que la musicoterapia tiene como principales objetivos, promover el bienestar, gestionar el estrés, aliviar el dolor, expresar sentimientos, mejorar la capacidad de memoria y consecuentemente la de aprendizaje, la adquisición de técnicas de comunicación, promover la coordinación, promover la rehabilitación física y promover comportamientos sociales.

En segundo lugar, encontramos las estrategias basadas en el tratamiento en la actividad física:

Una de las nuevas alternativas que se sugieren para mejorar la atención de niños y niñas con TDAH, es la intervención de actividad física dirigida. Se ha demostrado que, desde un punto de vista neuropsicológico, el ejercicio aeróbico tiene un impacto positivo sobre el cerebro, presentando beneficios a nivel cognitivos los que repercuten en las capacidades de aprendizaje y sociabilidad (Muñoz, et al., 2019, p. 43). 
Estudios afirman que el ejercicio aeróbico estimula los neurotransmisores aumentando sus niveles, que favorece la plasticidad sináptica, la velocidad de procesamiento cognitivo y el control inhibitorio lo que genera cambios en la estructura y función del cerebro humano (Gómez-Pinilla et al., 2008 como se cita en Muñoz, et al., 2019, p. 43; Bustamante, et al., 2019, p. 5).

Lomas y Clemente exponen que la práctica de actividad físico-deportiva podría producir beneficios en la función cognitiva, la función ejecutiva, la coordinación motora, el rendimiento académico, el comportamiento y las habilidades sociales del alumnado con TDAH (2017, p. 74)

Respecto a esta afirmación, se pueden evidenciar cambios en los resultados según la intensidad y la duración de la actividad física; el ejercicio aeróbico de intensidad moderada y de corta duración muestra mejoras de la concentración, la atención y el desempeño en ciertas tareas, sin embargo, una sola sesión de actividad física de corta duración no es suficiente para generar cambios prolongado (Bustamante, et al., 2019, p. 5).

Tras el análisis realizado por Bustamante, et al. (2019), estos concluyen que no hay evidencia definitiva que asegure que la actividad física es un tratamiento eficaz contra el TDAH, sí han demostrado que el ejercicio físico ayuda a complementar la terapia farmacológica, mostrando mejorar en las destrezas fundamentales de movimientos y la concentración (Bustamante, et al., 2019, p. 18).

En las investigación realizada por Muñoz (2019), se realizaron intervenciones deportivas durante 6 semanas, 2 veces por semana, las que tuvieron una duración de 1 hora aproximadamente, este tiempo dividido en un calentamiento inicial de 10 minutos seguidos de la realización de juegos aeróbicos durante unos 30 minutos y vuelta a la calma aproximadamente 5 minutos (Muñoz, et al., 2019, p. 40), como resultados de esta investigación se obtuvieron efectos positivos en las intervenciones en ejercicio físico aeróbico como complemento terapéutico de pacientes con trastornos cognitivos. Dentro de sus conclusiones afirman que:

La actividad física mejora la atención en niños y niñas con trastorno por déficit de atención con hiperactividad (TDAH). La intervención deportiva dirigida en el ámbito escolar podría ser un tratamiento complementario al farmacológico en pacientes con problemas de atención. Aumentar las horas de actividad física en el colegio y contar con profesionales del deporte que dirijan estas actividades puede mejorar la evolución clínica de pacientes con TDAH. (Muñoz, et al. 2019, p. 44)

Al efectuar ejercicio aeróbico estimula la proteína BDNF, un neurotransmisor que favorece la plasticidad sináptica lo que implicaría cambios en la estructura y función del cerebro humano, además de aumentar el tamaño del hipocampo y mejora la memoria (Erikson et al., 2011, Gómez-Pinilla et al., 2008 citados en Muñoz, et al. 2019). por lo que las investigaciones confirman que el desarrollo de actividad física dentro de los espacios escolares puede servir como parte del tratamiento para los estudiantes con TDAH. 
En tercer lugar, encontramos las estrategias basadas en las tecnologías de información y comunicación (tic's):

En la actualidad las tecnologías forman parte de nuestra vida, por lo que incorporarlas como estrategias educativas en nuestras salas de clases podría beneficiar a nuestros estudiantes, promoviendo el trabajo activo, colaborativo e interactivo de educadores y educandos (Fernández, Morillo, Gilibert, Carvalho, y Bello, 2020, p. 69).

Hoy podemos encontrar diferentes aplicaciones que pueden acompañar la intervención y/o tratamiento de estudiantes con TDAH como:

Sincrolab: Es una aplicación (app) utilizada como complemento terapéutico, que a través del juego diseña un entrenamiento especializado y dirigido en la recuperación y el desarrollo de capacidades cognitivas. Gracias al gran nivel de tecnología, a través de la inteligencia artificial esta app genera un programa adaptado al perfil del usuario y sus avances. Sincrolab es una herramienta de innovación, es aplicable tanto en un grupo pequeño como a nivel escolar, es una herramienta que sirve de apoyo a profesionales de la salud y educativos, es este quien entrega un usuario para que el estudiante o paciente, comience con el entrenamiento cognitivo.

Esta aplicación se encuentra tanto en la play store y google play, en dos versiones, una para niños y otra para adultos, además de incorporar una app para el profesional encargado, donde se visualizan los avances y las estadísticas de cada estudiante o paciente.

Una de las cosas para tener en cuenta a la hora de querer utilizar esta app es el costo, el que depende del uso que como profesional se le quiera dar a la plataforma. En su página web $^{1}$, podemos encontrar información de sus plataformas, así como investigaciones que demuestran la eficacia de la app.

Psious: Este software se basa en la implementación de tratamiento de exposición inmersiva virtual, combinada con técnicas psicoterapéuticas con el objetivo de reducir la activación fisiológica y modificar los pensamientos desadaptativos. Se presentan diversos escenarios, como entornos de realidad virtual y aumentada, vídeos en 3D y 360ㅜ, etc., empleados para el tratamiento de diferentes tipos de trastornos, especialmente para trastornos de ansiedad o fobias, pero se ha incursionado como apoyo para el tratamiento de TDAH como una práctica del mindfulness y técnicas de relajación (Fernández et al., 2020, p. 69). Facilita y potencia la inducción de emociones, pensamientos, reacciones similares obtenidas del paciente a una exposición real a ciertas situaciones o estímulos. Esta plataforma es utilizada por psicólogos y psiquiatras, para su utilización se requiere de un computador, un smartphone y lentes de realidad virtual. Este programa permite al paciente interactuar en entornos virtuales, situados entre la exposición real y la exposición imaginada.

\footnotetext{
${ }^{1}$ www.sincrolab.es
} 
Es el profesional quien tiene el control de las imágenes o situación a las que se expondrá al paciente, y el programa entrega un registro de las respuestas fisiológicas del paciente mediante biofeedback.

Al finalizar cada sesión, el sistema elabora un informe que permite al paciente ver sus progresos, con el objetivo de generar confianza y compromiso en la terapia (Fernández et al., 2020, p. 70).

El profesional que obtenga el software tendrá acceso a todo lo que hay en él, como manuales, videos tutoriales, descargas, sesiones formativas online, reuniones de expertos, cursos de formación certificados, etc., así como también a más de 70 actividades de realidad virtual, realidad aumentada y videos 360으 (Fernández et al., 2020, p. 70).

\section{METODOLOGÍA}

La investigación se basó en una metodología de carácter cualitativo descriptivo, ya que se fundamenta bajo el paradigma constructivista. Se realizó una minuciosa revisión bibliográfica para recopilar la información, que fue extraída de investigaciones anteriores y de la evolución de las teorías, hipótesis y hechos allí descritos. La revisión bibliográfica consiste en el análisis y selección de fuentes con el propósito de categorizar adecuadamente la relevancia del contenido de cada documento, ya que es la base para la toma de decisiones, formulación de conclusiones y comentarios en relación con la información recolectada del análisis total (Gómez-Luna et al, 2014). Para llegar a encontrar las fuentes, se utilizaron buscadores de datos de diferente índole, destacamos los más usados: Dialnet, Redalyc, Scielo y Google Académico.

A raíz de las mencionadas bases de datos, se establecieron filtros para la búsqueda, con la finalidad de obtener información más precisa para lograr responder a los objetivos planteados en la presente investigación. Los criterios de inclusión en la búsqueda fueron: Investigaciones inferiores a 10 años de publicación del año actual/ Investigaciones referidas al diagnóstico del TDAH/ Investigaciones enfocadas en estudiantes de enseñanza básica diagnosticados con TDAH/ Investigaciones referidas a las estrategias utilizadas con alumnos con TDAH y que cursen la enseñanza básica.

Añadiendo a la elección de material de investigación, se consideraron los siguientes criterios de exclusión: Investigaciones enfocadas en estudiantes de otro ciclo escolar/ Investigaciones referidas a otros trastornos o necesidades educativas. 


\section{RESULTADOS Y ANÁLISIS}

El TDAH es un trastorno de gran incidencia a nivel mundial, siendo su evaluación y diagnóstico un proceso principalmente clínico, estos diagnósticos son realizados por especialistas médicos de la salud, ya que el TDAH presenta un origen neurológico, el que tiene afectaciones en las funciones ejecutivas, lo que puede generar fracaso escolar durante los primeros años de estudios.

Para que este proceso se valide, luego de observar síntomas en los estudiantes, se comienza con el proceso de evaluación, con la obtención de información familiar y contextual del estudiante, que se levanta a raíz de entrevistas y test, que posteriormente son evaluados por los establecimientos educacionales, los que dependen de las normativas de cada país. Además de ello los docentes de los alumnos, responden a evaluaciones de los estudiantes para complementar la información entregada por la familia.

En consecuencia, del diagnóstico se realiza una serie de adecuaciones a los estudiantes en el aula para que estos puedan incluirse de la mejor manera posible dentro de ella, para esto enfrentan una serie de tratamientos, siendo la medicación de los alumnos el más utilizado.

A continuación, se presenta un cuadro resumen en donde se detalla a grandes rasgos las ventajas y desventajas de cada estrategia de intervención descrita en la revisión bibliográfica:

Tabla 1.

Estrategias de atención al TDAH

\begin{tabular}{|c|c|c|}
\hline Estrategia & Beneficios & Desventajas \\
\hline $\begin{array}{l}\text { Estrategias } \\
\text { tradicionales }\end{array}$ & $\begin{array}{l}\text { - Son fáciles de implementar. } \\
\text { - Requieren bajo costo. } \\
\text { - Son aplicables en cualquier } \\
\text { contexto. } \\
\text { - No requieren de una mayor } \\
\text { capacitación. } \\
\text { - Buscan prevenir las conductas. }\end{array}$ & $\begin{array}{l}\text { - No son individualizadas a cada } \\
\text { estudiante. } \\
\text { - Generan un mínimo impacto } \\
\text { en la conducta del estudiante. }\end{array}$ \\
\hline $\begin{array}{l}\text { Tratamiento } \\
\text { farmacológico }\end{array}$ & $\begin{array}{l}\text { - Apuntada a la regulación. } \\
\text { neurológica, principalmente de la } \\
\text { dopamina. } \\
\text { - Producir efectos emocionales de } \\
\text { alivio. } \\
\text { - Mejora del rendimiento escolar } \\
\text { mejoran su capacidad para } \\
\text { concentrarse. } \\
\text { - Reducen la hiperactividad y la } \\
\text { impulsividad. }\end{array}$ & $\begin{array}{l}\text { El uso del medicamento puede } \\
\text { generar: } \\
\text { - Experiencias de miedo. } \\
\text { - Efectos negativos sobre la } \\
\text { autoestima y el sentido de } \\
\text { control sobre la propia vida. } \\
\text { - Pérdida del sentido de } \\
\text { autonomía de los alumnos. }\end{array}$ \\
\hline
\end{tabular}




\begin{tabular}{|c|c|c|}
\hline Estrategia & Beneficios & Desventajas \\
\hline $\begin{array}{l}\text { Equinoterapia: } \\
\text { Horse emotion }\end{array}$ & $\begin{array}{l}\text { - Entorno que activa estímulos } \\
\text { sensoriales. } \\
\text { - Potencia habilidades } \\
\text { sensoriales. } \\
\text { - Responde a necesidades de } \\
\text { autoestima. } \\
\text { - Integra al estudiante al contexto } \\
\text { de la hípica. } \\
\text { - Fuera del ambiente escolar. }\end{array}$ & $\begin{array}{l}\text { - Se necesita personal } \\
\text { competente. } \\
\text { - Requerimiento de animales } \\
\text { entrenados. } \\
\text { - Se precisa de un ambiente } \\
\text { adecuado para la terapia. } \\
\text { - De alto costo. }\end{array}$ \\
\hline Mindfulness & $\begin{array}{l}\text { - Reduce el estrés. } \\
\text { - Optimiza el aprendizaje. } \\
\text { - Beneficia relaciones } \\
\text { interpersonales. } \\
\text { - Mejora la auto-regulación } \\
\text { emocional. } \\
\text { - Favorece la atención y } \\
\text { disposición del alumno. }\end{array}$ & $\begin{array}{l}\text { - Requiere de personal } \\
\text { competente. } \\
\text { - Se necesita capacitación para } \\
\text { la intervención. } \\
\text { - Costo medio. }\end{array}$ \\
\hline Musicoterapia & $\begin{array}{l}\text { - Promueve el bienestar. } \\
\text { - Mejora la capacidad de } \\
\text { memoria. } \\
\text { - Beneficia la capacidad de } \\
\text { aprendizaje. } \\
\text { - Potencia la expresión de los } \\
\text { sentimientos. }\end{array}$ & $\begin{array}{l}\text { - Precisa capacitación para la } \\
\text { intervención. } \\
\text { - Se necesita personal } \\
\text { competente. } \\
\text { - Requiere material. } \\
\text { - Costo medio. }\end{array}$ \\
\hline $\begin{array}{l}\text { Ejercicio } \\
\text { aeróbico }\end{array}$ & $\begin{array}{l}\text { - Las actividades físicas de } \\
\text { intensidad moderada y corta } \\
\text { duración, muestran mejoras en la } \\
\text { concentración y atención. } \\
\text { - Es un complemento a la terapia } \\
\text { farmacológica } \\
\text { - Mejora las destrezas } \\
\text { fundamentales del movimiento. } \\
\text { - Estimula los neurotransmisores } \\
\text { - Favorece la plasticidad } \\
\text { sináptica, la velocidad de } \\
\text { procesamiento cognitivo y el } \\
\text { control inhibitorio. }\end{array}$ & $\begin{array}{l}\text { - Requiere de intervenciones } \\
\text { constantes y sistemáticas. } \\
\text { - Es una estrategia que precisa } \\
\text { la implementación de otra } \\
\text { estrategia. }\end{array}$ \\
\hline Sincrolab & $\begin{array}{l}\text { - Es una app enfocada en el juego. } \\
\text { - Es un entrenamiento } \\
\text { especializado, ya que se adapta al } \\
\text { perfil de cada usuario. } \\
\text { - Es fácil de usar. }\end{array}$ & $\begin{array}{l}\text { - El alto costo de la app, que } \\
\text { varía según la cantidad de } \\
\text { estudiantes conectados. }\end{array}$ \\
\hline
\end{tabular}




\begin{tabular}{|c|c|c|}
\hline Estrategia & Beneficios & Desventajas \\
\hline Sincrolab & $\begin{array}{l}\text { - Está enfocado en la } \\
\text { recuperación y desarrollo de las } \\
\text { capacidades cognitivas. } \\
\text { - Tiene } 2 \text { versiones para niños y } \\
\text { para adultos, la cual está } \\
\text { adaptada a cada grupo. }\end{array}$ & $\begin{array}{l}\text { - El requerimiento de material } \\
\text { tecnológico, ya que el } \\
\text { estudiante debe contar con } \\
\text { acceso de celular o tablet. }\end{array}$ \\
\hline Psious: & $\begin{array}{l}\text { - Presenta gran versatilidad, ya } \\
\text { que es útil para diferentes } \\
\text { trastornos. } \\
\text { - Contiene más de } 70 \text { actividades } \\
\text { de realidad virtual, realidad } \\
\text { aumentada y videos } 360^{\circ} \\
\text { - El sistema elabora un informe } \\
\text { luego de cada intervención, para } \\
\text { que paciente y el profesional } \\
\text { conozcan sus avances, generando } \\
\text { confianza y compromiso en la } \\
\text { terapia. } \\
\text { - Realiza un registro de las } \\
\text { respuestas fisiológica del } \\
\text { paciente mediante biofeedback }\end{array}$ & $\begin{array}{l}\text { - Esta plataforma debe ser } \\
\text { utilizada por psicólogos y } \\
\text { psiquiatras. } \\
\text { - El alto costo de la plataforma. } \\
\text { - El alto costo del material } \\
\text { necesario, las gafas de realidad } \\
\text { virtual y los sensores } \\
\text { biofeedback. }\end{array}$ \\
\hline
\end{tabular}

Fuente: Elaboración propia.

Con los avances en la tecnología, se han ido desarrollando diversas herramientas de comunicación y desarrollo en este ámbito, así también ha sucedido en la educación, en la que han surgido software, aplicaciones y elementos tecnológicos que facilitan el trabajo con los estudiantes, como lo son las aplicaciones Sincrolab y Psious, que se han desarrollado para mediar con estudiantes con TDAH, las cuales pese a todos sus beneficios, aún son de alto costo y por tanto poco accesibles a considerar implementar en el contexto chileno.

También han ido surgiendo intervenciones alternativas a la medicación para tratar el TDAH, investigaciones revelan resultados positivos con el uso de terapias como lo son la musicoterapia, la práctica del mindfulness, la equinoterapia, el ejercicio aeróbico, además de la aplicación de apoyo conductual positivo. 


\section{CONCLUSIONES}

El sistema educativo chileno establece un proceso clínico para validar el diagnóstico de estudiantes con TDAH, el cual es sustentado por su origen neurológico el que requiere de una evaluación médica.

Por otra parte, en los establecimientos educacionales existe un levantamiento de información a través de diversos tests o encuestas a la familia y su contexto para realizar una anamnesis de los estudiantes y así determinar el diagnóstico más preciso.

Del diagnóstico de los estudiantes con TDAH se desprenden diversos tratamientos, algunos de ellos alternativos a los medicamentos, pero se evidencia que la medicación de los alumnos continúa siendo el más utilizado.

Hemos podido observar tras la investigación realizada, que se han incorporado nuevas formas de intervención para alumnos de TDAH, aun así, podemos encontrar estrategias tradicionales las cuales se utilizan en las escuelas chilenas. A nivel internacional, se desarrollan investigaciones cuyos objetivos apuntan a nuevas estrategias para intervención en estudiantes con TDAH, dentro de este panorama podemos ejemplificar los estudios realizados en España, allí se encuentran agrupaciones sociales que buscan ayudar a estos estudiantes, desarrollando en la actualidad herramientas tecnológicas, como lo es sincrolab.

Uno de los temas controversiales en estos últimos años, es el uso de medicamento como uno de los principales tratamientos a nivel escolar, pero debemos tener claro que este trastorno aún es un trastorno médico por lo que el uso del medicamento puede beneficiar a estos alumnos, pero éste no debe ser la única solución como tratamiento, ya que se deben implementar estrategias alternativas e interdisciplinarias que nos permita apoyar a los estudiantes con TDAH, tanto el área académica, como emocional y social, permitiéndonos sobrellevar de la mejor forma posible esta condición.

El TDAH es un trastorno heterogéneo, el cual no presenta la misma característica en todas las personas, por lo que no todas las estrategias servirán de la misma forma a cada sujeto. Sabemos que es un trastorno, que afecta las funciones ejecutivas, pero no en todos los casos afectan las mismas o de la misma intensidad, por lo que a la hora de tomar la decisión de las estrategias utilizadas para el estudiante, éstas deben ser orientadas a sus características personales así como también las posibilidades y características del contexto escolar, ya que el uso de estas estrategias podrían traer costos adicionales a los centros educativos, que no todos están dispuestos a asumir, como nuevas tecnologías y profesionales habilitados.

Es necesario, que las familias de estos estudiantes con TDAH, les consulten a los centros escolares, sobre las nuevas tecnologías y terapias complementarias con las que disponen para apoyar el proceso educativo del alumno y así tomar una decisión informada a la hora de matricular o no a sus hijos dentro de un centro específico que no tenga incorporado 
en proyecto educativo apoyos alternativos y complementarios a la medicación frecuentemente utilizada.

Las estrategias pueden ser muy diversas y la que pueden ser utilizada no sólo para niños con TDAH, sino que, en el contexto general de una escuela, ya que sabemos que trabajar la conducta puede ser beneficioso a nivel escolar al igual que el trabajo de las funciones ejecutivas, cómo va a ser el aprendizaje y que el estado emocional afecta en gran medida el aprendizaje de nuestros estudiantes.

Podemos concluir que el tratamiento de TDAH es visto desde una mirada clínica más que desde una mirada pedagógica, lo que ocasiona sobrediagnóstico y medicación, pero no soluciona el problema de fondo hacia el estudiante.

Finalmente, se puede concluir como hallazgo, que, al complementar el tratamiento de los estudiantes con estrategias didácticas innovadoras, alternativas y basadas en la emocionalidad y las tecnologías, pueden aumentar los resultados de las intervenciones con fármacos, o incluso sin ellos, dependiendo del sujeto. La complementariedad de estrategias, son aún mejores y favorables para los estudiantes, además es necesario tener el apoyo social y familiar para que logren sus objetivos educativos y de inserción social. 


\section{REFERENCIAS BIBLIOGRÁFICAS}

American Psychiatric Association. (2013). Diagnostic and Statistical Manual of Mental Disorders (5a ed.) (DSM-V). Washington DC (EEUU): APA. Recuperado de https://www.ncbi.nlm.nih.gov/pmc/articles/PMC3777342/

Acebes, A. y Carabias, D. (2014) El alumnado de primaria con Trastorno por Déficit de Atención/Hiperactividad (TDAH): la Musicoterapia como herramienta integradora dentro del contexto del aula de música. Revista Electrónica de LEEME. Number 38, pp. 1-16. https://ojs.uv.es/index.php/LEEME/article/download/9881/9299

Balbuena, F., Barrio, E., Gonzalez, C., Pedrosa, B., Rodríguez, C., \& Yaguez, L. (2014). Protocolo para la detección y evaluación del alumnado con trastorno por déficit de atención e hiperactividad en el ámbito educativo. Guía para orientadores y orientadoras. Recuperado de http://feaadah.org/docdow.php?id=581

Barceló, F. y Longo, D. (2018). Reflexiones acerca del Trastorno y Diagnóstico del TDA/TDAH, una mirada desde la clínica y social en nuestra actualidad. $X$ Congreso Internacional de Investigación y Práctica Profesional en Psicología XXV Jornadas de Investigación XIV Encuentro de Investigadores en Psicología del MERCOSUR. Facultad de Psicología Universidad de Buenos Aires, Buenos Aires.

Bustamante E., Santiago M., Ramer J., Balbim G., Mehta T., \& Frazier S. (2019). Actividad física y tdah: evidencia sobre el desarrollo, efectos neurocognitivos a corto y largo plazo y sus aplicaciones. Pensar en Movimiento: Revista de ciencias del ejercicio y la salud, 17(1), 32-59. Recuperado de https://www.scielo.sa.cr/pdf/pem/v17n1/1659-4436pensarmov-17-1-00032.pdf

Eraud, A. (2020). Una propuesta de intervención de equinoterapia para niños con TDAH desde una perspectiva de protección animal. dA. Derecho Animal. (Forum of Animal Law Studies) vol. 11/2. pág. 107, 125. Recuperado de https://doi.org/10.5565/rev/da.495

Fernández, M., Morillo, M., Gilibert, N., Carvalho, C., \& Bello, S. (2020). Herramientas tecnológicas del diagnóstico y tratamiento del trastorno por déficit de atención $e$ hiperactividad. Medicina (Buenos Aires), 80 (Supl. 2), 67-71. Recuperado en 02 de noviembre 2020, 2020, de http://www.scielo.org.ar/scielo.php?script=sci_arttext\&pid=S002576802020000200015\&lng=es\&tlng=es.

Holz, M. (2016). Datos de la modalidad de Educación Especial en Chile, año 2018. Asesoría Técnica Parlamentaria, 1-5. Recuperado de https://obtienearchivo.bcn.cl/obtienearchivo?id=repositorio/10221/26781/2/BCN _datos_de_EE_y_estudiantes_con_NEE_Final.pdf 
Huguet, A. y Alda, J. (2019). Mindfulness for Health. Programa de mindfulness para niños con TDAH Barcelona: Hospital Sant Joan de Déu (ed). https://www.sjdhospitalbarcelona.org/ y http://faros.hsjdbcn.org

Lomas, A. y Clemente, A. (2017) Beneficios de la actividad físico-deportiva en niños y niñas con TDAH. Revista Digital de Educación Física. Año 8, Núm. 44. https://dialnet.unirioja.es/servlet/articulo?codigo $=5807535$

López, I. y Pastor, S. (2019). Mindfulness y el Abordaje del TDAH en el Contexto Educativo. Revista Brasileira de Educação Especial. vol. 25. p. 175-188. Recuperado de: https://www.scielo.br/scielo.php?pid=S141365382019000100175\&script=sci_arttext

MINEDUC. Ministerio de Educación de Chile. (2015). Decreto 83/2015, que aprueba criterios y orientaciones de adecuación curricular para estudiantes con necesidades educativas especiales de educación parvularia y educación básica. Recuperado de https://especial.mineduc.cl/wp-content/uploads/sites/31/2016/08/Decreto-832015.pdf

MINEDUC. Ministerio de Educación de Chile. (2009). Decreto 170/2009, que Fija normas para determinar los alumnos con necesidades educativas especiales que serán beneficiarios de las subvenciones para educación especial. Recuperado de https://especial.mineduc.cl/wp-content/uploads/sites/31/2018/06/DT0-170_21ABR-2010.pdf

MINEDUC. Ministerio de Educación de Chile. (2016). Manual de apoyo a sostenedores y establecimientos educacionales para la implementación del Programa de Integración Escolar (PIE), en el marco de la Inclusión. Recuperado de: https://especial.mineduc.cl/wp-content/uploads/sites/31/2017/12/ManualPIE.pdf

MINEDUC. Ministerio de Educación de Chile. (2018). Instructivo de Incorporación de estudiantes al Programa de Integración Escolar. Recuperado de https://especial.mineduc.cl/wpcontent/uploads/sites/31/2017/12/INSTRUCTIVO-PIE_2018.pdf

Muñoz, D.; Díaz, A.; Navarro, J.; Camacho, P.; Robles, A.; Ibáñez, M.; Coronilla, M.; Gil, E.; Carballar, A.; Cano, R. (2019). Mejora de la atención en niños y niñas con tdah tras una intervención física deportiva dirigida. Cuadernos de Psicología del Deporte, 19(3), 3746. Recuperado de http://scielo.isciii.es/pdf/cpd/v19n3/1578-8423-CPD-19-300037.pdf

National Collaborating Centre for Mental Health UK (2009) Attention Deficit Hyperactivity Disorder: Diagnosis and Management of ADHD in Children, Young People and Adults. Leicester (UK): British Psychological Society (UK). PMID: 22420012. 
National Institute of Mental Health (2016) El trastorno de déficit de atención con hiperactividad: información básica. Recuperado 15 Octubre 2020, de https://www.nimh.nih.gov/health/publications/espanol/trastorno-de-deficit-deatencion-con-hiperactividad-informacion-basica/index.shtml

Peña Olvera, F., Palacio Ortiz, J. D., \& Barragán Pérez, E. (2010). Declaración de Cartagena para el Trastorno por Déficit de Atención con Hiperactividad (TDAH): rompiendo el estigma. Revista Ciencias De La Salud, 8(1). Recuperado de https://revistas.urosario.edu.co/index.php/revsalud/article/view/1285

Reyes, P, Cottet, P, Jimenez, A, \& Jauregui, G. (2019). Repensando la medicalización: posiciones discursivas de niños y de sus cuidadores sobre el diagnóstico y tratamiento del TDAH en Chile. Saúde e Sociedade, 28(1), 40-54. Recuperado de https://dx.doi.org/10.1590/s0104-12902019181141

Rodríguez, D. (2019) Conocimiento de los profesores sobre la musicoterapia en el proceso educativo de niños con TDAH Tesis de Grado. Universidad Nacional Federico Villarreal, Lima, Perú. Recuperado de http://repositorio.unfv.edu.pe/handle/UNFV/3142

Tenorio, S. (2011). Formación inicial docente y necesidades educativas especiales. Estudios pedagógicos (Valdivia), 37(2), 249-265. Recuperado de https://dx.doi.org/10.4067/S0718-07052011000200015

Varia, M., \& Kleinsteuber, K. (2014). Dificultad de aprendizaje en el niño. Revista Pediatría Electrónica, $\quad 11\left(\mathrm{~N}^{\circ} \quad 2\right), \quad 18-35 . \quad$ Recuperado de http://www.revistapediatria.cl/volumenes/2014/vol11num2/pdf/DIFICULTAD_AP RENDIZAJE_NINO.pdf 